\title{
Dietary intervention is effective in reducing the glycaemic index of maternal diets during pregnancy
}

\author{
C. McGowan, S. Levis, S. Curran, C. Toher, J. Byrne, M. Foley, R. Mahony and F. McAuliffe \\ School of Medicine and Medical Science, National Maternity Hospital, University College Dublin, Dublin, \\ Republic of Ireland
}

Recent trends have shown that infant birth weight in Ireland has increased over the past number of years ${ }^{(1)}$, implying that environmental factors may be partly responsible for this increase. It has been suggested that consuming a low-glycaemic index (GI) diet during pregnancy may help to reduce the risk of macrosomia by influencing maternal blood glucose concentrations, hence regulating delivery of glucose to the foetus $^{(2)}$. The aim of the present study was to investigate the impact of early pregnancy dietary intervention on the mean GI of maternal diets during pregnancy.

A subset of 123 secundigravid women from an ongoing large randomised controlled trial was included in the analysis. None of the participants were taking regular medication and none had diabetes or a history of gestational diabetes. On recruitment fifty-four participants were randomised to receive dietary advice and sixty-nine received no dietary intervention. Women in the intervention group received advice from a dietitian in groups of two to six individuals on one occasion between 10 and 18 weeks of gestation. This advice included recommendations for following a low-GI diet as well as overall healthy eating guidelines for pregnancy. All participants completed $3 \mathrm{~d}$ food diaries during their first trimester (baseline) and during both the second and third trimesters. It was requested that the women record in as much detail as possible their food and beverage intakes. Collected data were entered into NetWISP version 3.0 (Tinuviel Software, Llanfechell, Anglesey, UK). New food codes were created where necessary and many GI values were added or amended using the most up-to-date and relevant GI data from UK and international sources ${ }^{(3)}$. Statistical analysis was carried out using SPSS version 14.0 (SPSS Inc., Chicago, IL, USA) and differences in mean dietary GI between the groups were examined using independent $t$ tests. Changes were considered significant at $P<0.05$.

At baseline there was no significant difference in dietary GI between the intervention and control groups; however, following dietary intervention mean GI was significantly lower among the intervention group, as illustrated in the Table.

\begin{tabular}{|c|c|c|c|c|c|}
\hline & \multicolumn{2}{|c|}{ Intervention group $(n$ 54) } & \multicolumn{2}{|c|}{ Control group ( $n$ 69) } & \multirow[b]{2}{*}{$P$} \\
\hline & Mean & $\mathrm{SD}$ & Mean & $\mathrm{SD}$ & \\
\hline 1st Trimester & 51.2 & 6.67 & 51.8 & 6.15 & 0.551 \\
\hline 2nd Trimester & 50.5 & 6.67 & 52.9 & 5.88 & 0.031 \\
\hline 3rd Trimester & 49.9 & 5.84 & 52.8 & 6.85 & 0.008 \\
\hline
\end{tabular}

Results indicate that dietary advice delivered in a small group context is effective in reducing the mean dietary GI of pregnant women. Dietetic input had a positive influence on patient behaviour, as it would appear that there was a high level of compliance to dietary advice among this patient group. This finding highlights the value of dietary advice and is an important consideration in relation to future research into associations between diet and pregnancy outcomes.

1. Mahony R \& O'Herlihy C (2003) Irish Med J 96, 88-89.

2. Moses RG, Luebcke M, Davis WS et al. (2006) Am J Clin Nutr 84, 807-812.

3. Atkinson FS, Foster-Powell K \& Brand-Miller JC (2008) Diab Care 43, 469-470. 